\title{
NOTAS PARA UNA HISTORIA DE LAS REIVINDICACIONES FEMINISTAS EN Y TRAS MAYO DE 1968: CONTRADICCIONES, ALIANZAS Y DESAFÍOS
}

\author{
NOTES FOR A HISTORY OF FEMINIST DEMANDS IN AND AFTER MAY 1968: \\ CONTRADICTIONS, ALLIANCES AND CHALLENGES
}

\author{
Elisa Cabrera García \\ Universidad de Granada \\ Irene Valle Corpas \\ Universidad de Granada
}

\section{RESUMEN}

Este artículo está pensado como un pequeño recorrido histórico que, partiendo del Mayo francés y arribando en el presente, señale la tendencia del feminismo a aliarse con otros impulsos anticapitalistas. Analizaremos primero las flagrantes contradicciones que marcaron el acontecimiento parisino notando, no obstante, en qué medida supuso el pistoletazo de salida para un feminismo radical e interseccional. Seguidamente comentaremos cómo en la década de los ochenta y noventa, y dado un giro neoliberal tanto en la economía como en el discurso, asistimos al olvido de anteriores líneas de denuncia. Tal repaso histórico debería permitirnos comprender las razones que motivan una actual relectura de textos clásicos del feminismo radical y de los desafíos a los que el movimiento se enfrenta a la hora de rehacer un enfoque unitario.

Palabras Clave: Mayo 68, Feminismo, Neoliberalismo, Cinzia Arruzza, Nancy Fraser, interseccionalidad, trabajos reproductivos.

\section{ABSTRACT}

This article is intended as a short historical journey from May in France to the present, pointing out the tendency of feminism to ally with other anti-capitalist impulses. We will first analyze the blatant contradictions that marked the Parisian event, noting, however, to what extent it was the starting moment for a radical and intersectional feminism. In addition to this we will comment on how in the 1980s and 1990s, within a neo-liberal turn in both economics and discourse, we witnessed the forgetfulness of previous lines of subversion. Such a historical review should enable us to understand the reasons for a current re-reading of the classic texts of radical feminism and the challenges that the movement faces in rebuilding a unitary approach. 
Keywords: May 68, Feminism, Neoliberalism, Cinzia Arruzza, Nancy Fraser, intersectionality, reproductive work.

\section{SUMARIO}

1.- Introducción. 2.- Mirar de cerca (las contradicciones). Mayo del 68 y las mujeres. 3.- Los años setenta y ochenta. Entre la interseccionalidad y la identidad. 4.- ¿Éxito o precariedad? El feminismo ante neoliberalismo. 5.- Desafíos actuales. Feminismo poscrisis y recuperación de las formas políticas del feminismo de los setenta. 6.- Bibliografía.

\section{Introducción}

Lo sabemos, nos ha sido dicho una y mil veces: en Mayo de 1968 en las calles de París se hablaba de amor y deseo. En aquella primavera fulgurante no se discutió únicamente sobre condiciones laborales, las ocupaciones de fábricas o la última jugada del grupúsculo enemigo. No sólo de la lucha política, también se habló de placer -o, con frecuencia, se pregonó la fusión de ambos impulsos- y abiertamente de sexo. A falta de otras pruebas nos lo confirman las pintadas en las paredes: «iii Te amo!!! ¡Oh, díganlo con adoquines!»; «Desabrochen el cerebro tan a menudo como la bragueta»; "Cuánto más hago el amor, más ganas tengo de hacer la revolución. Cuánto más hago la revolución, más ganas tengo de hacer el amor». Ciertamente, junto a las demandas en favor de un verdadero acceso de las clases populares al poder, se urdían estas otras proclamas que pedían una vida liberada en una sociedad menos biempensante pero más relajada. Lo sabemos muy bien y no sólo porque efectivamente así ocurriese y nos haya llegado el recuerdo, sino sobre todo porque en nuestro imaginario actual tal episodio ha sido reducido a una enorme algarada para conquistar solo este tipo de ruegos.

Como ha advertido con amargura Kristin Ross, desde los años ochenta en adelante, dado el devenir histórico de Francia y por impacto de la Nouvelle Philosophie-un pensamiento escorado a la derecha del espectro político-, en las conmemoraciones de las revueltas, la temática de la revolución sexual es la prima donna del espectáculo. Los principios mayoritariamente marxistas que movilizaron a los estudiantes quedan limitados a la búsqueda de una mayor autonomía personal. Hasta se ha llegado a tildar lo sucedido en mayo y junio -que recordemos, fueron, entre otras cosas, la batalla campal contra la policía en el Barrio Latino y una huelga de nueve millones de trabajadores-, como la «gran revolución cultural liberal/libertaria» (Ross, 2008: 37). Aun celebrando tal renovación de las costumbres -de la que aún somos herederas- podemos decir, sin miedo alguno, que no entenderemos mucho ni de la contracultura ni de los distintos conflictos de la década, si los tomamos por una 
simple «revuelta de las braguetas»-recogiendo el mensaje del grafiti. O si los vemos, como se ha querido hacer en alguna otra ocasión, como un psicodrama familiar que enfrentaba a padres déspotas y anticuados con sus soñadores y enrabietados hijos y a amas de casa insatisfechas y desagradecidas con sus cansados maridos. Pero, lo que es aún más curioso es que esta reducción tan sospechosa deja de lado un elemento crucial: sí, en Mayo también se habló de sexualidad y amor -como se habló de todo- pero las voces solían ser masculinas. Y sin embargo, esto no debe llevarnos a zanjar el asunto. Si en Mayo se abordaron todas estas cuestiones es porque algunas de las tensiones que alberga la vida social y privada en el capitalismo desarrollista acabarían por restallar en aquellos años aguerridos. Y aunque a las mujeres no se les permitiese participar en los acontecimientos en igualdad de condiciones no podemos negar que puede y debe hacerse una lectura de género de esta larga onda de protestas que arroje algo de luz sobre la relación del 68 con las mujeres y sus luchas -y de estas con nuestro panorama actual-, sin caer otra vez en lugares comunes inoperantes. Tal es el ímpetu que mueve a este trabajo. Para ello, creemos necesario elaborar un pequeño relato histórico de las tres variantes teóricas, en tanto que modelos de comprensión de la relación entre el género y la clase, que se sucedieron desde entonces. Nuestro empeño será vislumbrar las distintas variables de la solidaridad del feminismo en su apoyo a otras corrientes críticas.

Como punto de partida, digamos, por ahora con Cinzia Arruzza, que el impulso que tomaron las luchas de las mujeres y su elaboración teórica no habría sido posible sin el contexto de agitación creado por el 68 y los movimientos que lo sucedieron (Arruza, 2015: 61), pero es precisamente a partir de la experiencia del 68 cuando se produce una escisión entre los estudiantes, los obreros y los colectivos de feministas. Mayo puede entenderse así como un verdadero impasse, pues supuso una divergencia tanto en lo que a las formas de representación se refiere como en las cuestiones que apremiaba reivindicar. A partir de esta separación post-sesentayochista y a lo largo de la década de los 80 , asistimos -entre otros escenarios- a la explosión del feminismo como catalizador de una serie de movimientos identitarios para, finalmente y en los últimos compases del siglo, presenciar la consolidación del feminismo neoliberal ya bajo las nuevas formas de producción y consumo posfordista. Pero, eso sí, no sin todos los gestos de desapruebo, mareas críticas y reacciones alternativas a tal deriva congruentes con una coyuntura de crisis como la actual.

\section{Mirar de cerca (las contradicciones). Mayo del 68 y las mujeres}

Cuando se vuelve al episodio de Mayo, ya sea en un acto conmemorativo o en un trabajo académico, suele recurrirse a los términos «crisis», «ruptura» y sobre todo «eclosión» 
-rara vez, por el contrario, se emplea la palabra «guerra». Podríamos preguntarnos entonces, ¿qué fue lo que entró en crisis, qué fue lo que sufrió una ruptura y qué eclosionó realmente en tan pocas semanas? Nada menos, creemos, que un nutrido conjunto de contradicciones que venían gestándose desde la inmediata posguerra. $Y$ con ellas apareció también una nueva hornada de pensadores y pensadoras -pues por primera vez fueron a la universidad una generación de mujeres- que se dotaron de los instrumentos teóricos convenientes para hacer frente a toda la madeja de contrasentidos de la que estaba tejida la vida en las sociedades del desarrollismo. Las cosas, pensaban, las cosas más evidentes, no habían sido estudiadas con la debida atención. Si hubo un bajo continuo que atravesó la variedad infinita de acercamientos en estos decenios de desenfreno teórico fue, sin duda, la exigencia de exploración cuidadosa de la realidad en todos sus matices. La teoría y la filosofía no iban a sentir, a partir de ahora, vergüenza alguna -antes bien entusiasmo- en preguntarse por las cosas más prosaicas aunque ello las indujese a adentrarse en el terreno de la política. Y la consigna era clara: había que mirar los fenómenos detenidamente para comprender que todo el horizonte cotidiano se sustentaba en un pacto inestable entre capital y trabajo y en la división sexual del mismo, a la que acompañaba la imposición de unas relaciones sociales, sexuales y de poder innegablemente clasistas y machistas. El heterodoxo pensamiento de los sesenta advirtió que la crisis era en realidad un haz de guerras, o lo que es lo mismo, supo observar que al sistema le saltaban las costuras por todos sitios. A finales de los sesenta explotó lo que Maurizzio Lazzarato y Éric Alliez han denominado un crack-up, un ataque a todos los elementos y jerarquías que dirigían los Estados y las sociedades:

No solo tenemos la historia del capital a través de la guerra, sino igualmente esta otra historia a través del 68 que hace posible el pasaje teórico de «la» guerra a «las» guerras. [...] Las guerras de subjetividad no son un «suplemento» del capital en su cara «subjetiva», sino que constituyen la especificidad más «objetiva» de las guerras impulsadas contra las mujeres, los locos, los pobres, los criminales, los jornaleros, los obreros, etcétera (Lazzarato y Alliez, 2017: 25-26, 58)'.

En esta guerra las mujeres tomaron parte en muchísimos aspectos antes de constituirse como movimiento compacto que daría pie a hablar de esa Segunda Ola del Feminismo. Durante los sesenta y principios de los setenta, las mujeres participaron en las protestas no con el perfil de una lucha separada que habría de pegarse como un ismo más, sino manteniendo un impulso de unión, esa tan cacareada unión que era la clave del 68. Las feministas pusieron

1 El nervio del argumento de estos investigadores -que se sirven, entre otras, de las aportaciones de Deleuze, Foucault, Schmitt o Federici- es que la acumulación primitiva, analizada por Marx en el capítulo 24 de El Capital, no se detiene sino que constituye un continuum de expropiación. 
sobre la mesa problemas que eran tan universales como los de sus compañeros hombres. En especial, extendieron el principio de que todo es producción, no sólo aquello que sale de las manos callosas de un operario industrial. Así reconocían, por su parte, Antonio Negri y Felix Guattari el valor de haber puesto en el ojo de mira problemas ahora tan pertinentes:

Antes de 1968 el problema de la reproducción era marginal respecto al de la producción. El movimiento de las mujeres lo ha convertido en central. Cuando los problemas relativos a la formación de la fuerza de trabajo abstracta e inmaterial parecían secundarios respecto a la fuerza de trabajo fabril, los movimientos de los estudiantes los han situado en un lugar central, al mismo nivel que las nuevas necesidades propuestas por la imaginación teórica y estética (Negri y Guattari, 1999: 35).

Sería por ello necesario estar atentos al hecho de que todo lugar podía acabar siendo una fábrica, desde el hogar al útero materno. Recordemos con Federici que la fuerza que tomaron las pensadoras feministas de los movimientos anticolonialistas y de estudiantes las llevó a:

ampliar el análisis marxista sobre el trabajo no asalariado más allá de los confines de las fábricas y, así, contemplar el hogar y el trabajo doméstico como los cimientos del sistema fabril más que como su «otro». [...] Desde nuestra perspectiva, a primera vista, resultó obvio que el circuito de la producción capitalista, y de la «fábrica social» que esta producía, empezaba y se asentaba primordialmente en la cocina, el dormitorio, el hogar -en tanto que estos son los centros de producción de la fuerza de trabajo- y que a partir de allí se trasladaba a la fábrica pasando antes por la escuela, la oficina o el laboratorio. En resumen, no acogimos pasivamente las lecciones de los movimientos que he señalado anteriormente, sino que los pusimos patas arriba, exponiendo sus límites, utilizando sus piedras angulares teóricas para construir un nuevo tipo de subjetividad política y de estrategia (Federici, 2013: 23-25).

Junto a esta amplitud de espacios ${ }^{2}$, las feministas también ayudaron a consolidar otro principio de extrema importancia, a saber, que todo es político -o lo que es lo mismo, todo está históricamente determinado- y por consiguiente las cuestiones privadas, psicológicas o sexuales no debían abordarse como hechos íntimos, naturales e inalterables, pues habían sido creadas por (y afectaban a) una comunidad. Las nociones mismas de naturaleza y de normalidad iban a caer por su propio peso. De hecho, hasta los fenómenos más aparentemente naturales como el nacimiento o la relación hombre-mujer-hijo serían objeto de pertinentes análisis políticos a fin de ser rediseñados en modos más liberadores para las mujeres. Si el

2 El auge del feminismo desencadenará importantes consecuencias a la hora de concebir y criticar el espacio urbano y social, su reparto y su producción. Cartografiar esta geografía de la desigualdad fue una tarea en la que se emplearon ensayistas como Doreen Massey o Gillien Rose. 
adagio no era otro que ese famoso «tout est politique», ello significa que la categoría mujer, como la categoría negro o pobre, era ante todo una cuestión política y nos atreveríamos a destacar que se trataba de una cuestión política cuya raíz, entendían, era económica, con lo cual el enemigo no sería otro que el mismo capitalismo contra el que gritaban sus compañeros. Sigamos el relato de Arruzza a modo de guía:

El nuevo feminismo se afirmó y se difundió por todo el planeta en esa intersección entre los movimientos estudiantiles y juveniles, el nuevo movimiento obrero, las luchas de liberación nacional, los movimientos antisegregacionistas, el Black Power, etc., que fueron el 68 y los años sesenta y setenta. [...] La contestación juvenil había puesto en cuestión el modelo social existente, criticando no solamente las relaciones de producción, sino también las relaciones sociales, los clichés y los estereotipos impuestos, el conformismo y la cultura del bienestar, el autoritarismo y las relaciones de poder difundidas. En esta radical puesta en cuestión de lo existente, en la alusión $a$, y en la tentativa de, poner en práctica relaciones distintas, las nuevas feministas encontraron los instrumentos de crítica con los que volverse contra la dominación masculina en la cultura, la sociedad, la producción, la política y la familia. [...] Ya no se contentarían con declarar la plena participación de las mujeres en la vida política y social. [...] Se inició por eso una crítica y una deconstrucción sistemática de las formas de la política y de la vida social, así como de la cultura, con el fin de mostrar su carácter patriarcal (Arruzza, 2015: 61).

El tourbillon social del 68 contribuyó a revelar los mecanismos de justificación del orden imperante y en los años subsiguientes el movimiento feminista supo recoger ese relevo de forma privilegiada en los futuros Movimientos de Liberación de la Mujer. Armadas con estos dos axiomas y apoyadas sobre este puñado amplio de alianzas, las activistas feministas estaban preparadas para empezar a quitarle el barniz a la realidad y proclamar un año cero, una nueva era en la que había que combatir las desigualdades desde todos los frentes. Y sin embargo, no todo parecía ser tan fácil ni tan evidente para ese «nuevo feminismo». Por supuesto que ni el Mayo francés ni el resto de actuaciones, corrientes y organizaciones parecían querer librarse del machismo y el heterosexismo. Basta con volver sobre el severo testimonio de personajes que, como Lionel Soukaz, sabían que para ellos el combate sería largo:

Mayo del 68 es sexista. El 1 de mayo de 1971, los Gazolines, detrás de la CGT, fueron insultados y golpeados. Los heterosexuales juegan al Che Guevara y por la noche, sus novias limpian a los niños, cocinan, mientras hacen la revolución. Los izquierdistas quieren la revolución y en realidad perpetúan la opresión -sobre las mujeres y los maricones. Esto es lo que cambiará con el surgimiento de los movimientos feministas. Los maricones añadimos esta idea: la revolución es también un sexo (Fleckinger y Neveux, 2007: 318 ). 
Y no sorprende, pues en la mayoría de los casos la tan cantada solidaridad no fue engarzada dejando de lado las peticiones de muchos, entre otros, la de las mujeres. Ese fue uno de sus más dolorosos fracasos. Es conocido y ha sido profundamente señalado y criticado el papel periférico que se les asignó a las mujeres en la revuelta de Mayo; en los diferentes comités de acción, en las Asambleas Generales de la Soborna, Nanterre o Censier. Es también sabido el uso sexualizado que se hizo de las imágenes del cuerpo femenino para generar un discurso ficticio y descafeinado de las revueltas. Este rol secundario se puede comprobar directa y abiertamente con un dato puramente objetivo que nos permite adentrarnos en la problemática. En su libro Mai retrouvé Jacques Baynac (2016) relata casi minuto a minuto lo acontecido en cada uno de los encuentros de Mayo. Salvo un puñado de intervenciones en asambleas que nunca derivan en nada más, en cuatrocientas páginas el lector sólo encuentra el relato de una mujer actuante, la profesora de secundaria Elisabeth Brünner, integrante del Comité de Acción Trabajadores-Estudiantes de Cersier. Esta protagonista efímera viaja a la provincia de Troyes para entablar contactos con trabajadores de las fábricas y, según la narración que nos hace Baynac, en el mismo momento en el que Brünner inicia su discurso ante el público obrero, no deja de preguntarse: «¿̇Por qué estudio historia? ¿Por qué soy profe? ¿̇Por qué no estoy en un grupo político?». No tardará en responderse a sí misma: «Porque soy mujer [e] intento adquirir vocabulario para imitar el estatus masculino» (257-260). Sin ir más lejos, Baynac en este mismo capítulo dedica un par de párrafos algo idealistas a la supuesta abolición de las antiguas relaciones entre hombres y mujeres en el marco de la revuelta: «Los chicos parecen estar esperando algo de las chicas. Ellas se sienten investidas de cierta responsabilidad. Se descubren capaces de tener iniciativas en todos los dominios. Y de la misma forma que gestionan las míseras economías del grupo [...] también deciden como todos los demás, sobre las acciones políticas» (258). Baynac parece no darse cuenta de la diferencia entre poder votar y poder proponer y parece incluso querernos decir que las voces de, como él mismo dirá, «chicas que se atrevieron a hablar, por fin» (258), lo hicieron a instancias de unos compañeros que, considerando que eran realmente válidas, les regalaron la oportunidad de hacerlo.

Además de tener que esperar pacientes a ser escuchadas, sabemos también que eran ellas las que gestionaban la economía y los víveres, organizaban la cocina y repartían las comidas dentro de las organizaciones. Hace apenas unos meses lo recordaba, no sin cierta amargura, la legendaria activista Angela Davis en su intervención en un congreso de conmemoración del 68: «We did the housework of the movement» ${ }^{3}$. Y se hace difícil para el

3 Véase el programa del Congreso Global '68//Solidarity in Alliance and Global History, París, 2-6 de Mayo de 2018. Disponible en http://www.fmsh.fr/en/college-etudesmondiales/29161 (31/08/2018). 
lector informado no recordar aquel personaje tan complejo como elocuente de la chica que limpia el piso en la controvertida película La chinoise del 67 de Jean-Luc Godard. Pareciera como si Godard no sólo hubiese preconizado el événement sino también hubiese vaticinado sus sesgos de género y clase. El papel que encarna Juliet Berto es el de una sirvienta llegada a la capital desde el campo que acaba prostituyéndose y limpiando los barrios ricos de París. Tras escapar a esos empleos miserables para las clases altas se integra en el cenáculo de estudiantes radicalizados. El problema es que también allí acaba fregando los platos y haciendo labores de servicio para estos jóvenes maoístas. ¡Ni tan siquiera una heterotopía instalada en un apartamento de marxistas díscolos estaba al resguardo del machismo! Allí estallarían las mismas contradicciones de género, de clase y de roles políticos dentro del trabajo revolucionario. Allí también las mujeres podían ser tratadas como figurantes secundarias, laboriosas asistentas o simplemente chicas guapas. La propia Elisabeth Brünner manifestará su desencanto ante este espejismo de igualdad al reflexionar: «Supe que mayo había terminado cuando, después del 24, un tipo se puso a ligar conmigo en la escalera de Cersier. Era la reinstauración del viejo orden. Y yo volvía a ser una tía» (258). Y lo que es peor aún, no eran casos aislados ni episodios desafortunados. Algunos movimientos parecían participar de un imaginario que sexualizaba desmedidamente el cuerpo femenino. Un buen ejemplo lo podemos ver en un montaje que realizó la Internacional Situacionista que, como señaló en su excelente monografía recientemente publicada De grands soirs en petits matins Ludivine Bantigny, "acostumbrados a pensar la reificación de las relaciones sociales y el fetichismo de la mercancía», utilizaron el cuerpo de una mujer exuberante, con sus pechos expuestos, que rezaba: «Ah... la Internacional Situacionista...» (Bantigny, 2018: 261).

Junto al trato desfavorable de ciertos sectores de la izquierda, durante los acontecimientos, las mujeres también hubieron de sufrir los abusos de las autoridades. Algunas de las que participaron en las protestas sufrieron una violencia policial machista y específicamente sexual. El libro negro de las jornadas de mayo menciona cómo, en numerosas ocasiones, la policía trataba a las manifestantes de «putains dont ils allaient s'occuper» [putas de las que habría que ocuparse], $\mathrm{u}$ otras expresiones tan denigrantes como: «Espèce de salope, on va te faire défiler dans les rues de Paris à poil» [Pedazo de guarra, te vamos a hacer desfilar por las calles de París en pelotas]. (Bantigny, 2018: 261). Si bien, y a pesar de la gravedad de las ofensas, según Bantigny, en el clima de agitación no hubo ocasión para pensar o criticar este comportamiento de la policía reseñando su naturaleza eminentemente sexista. Pero las mujeres no tardarían en poner el grito en el cielo no sólo contra este tipo de prácticas sino en términos más generales contra el funcionamiento de las instituciones. Para ello desarrollaron un análisis y una denuncia de las violencias sexuales y las violaciones a mujeres también 
en el terreno jurídico. El trabajo de la socióloga Jean Bérard (2014) ha mostrado que la contradicción que se vivió en el mismo movimiento y en los años inmediatamente posteriores al 68 hizo vascular las posturas entre aquellas que señalaban la necesidad de tipificar penalmente violencias sexuales tan graves como la violación y el movimiento de liberación de las prisiones -integrado por maoístas y trotskistas-, que pretendía crear algo así como una «justicia popular» alternativa. Es decir, las mujeres activistas sostuvieron que el sistema penal precisaba ser abordado con urgencia desde una perspectiva feminista y dentro de una crítica radical de las instituciones.

A pesar de este panorama nada alentador, decíamos antes que sin el gran terremoto de aquella primavera, que movió todas las aguas mansas, hubiese sido difícil pensar la gran explosión posterior del feminismo. Sí, Mayo del 68 reprodujo socialmente los roles de género pero las propias participantes del movimiento no quisieron aceptar esa asignación. Siguiendo este espíritu, Arruzza ha notado que aunque la voz, opiniones o ideas de las mujeres apenas tuvieron ninguna relevancia en los comités de acción y en las Asambleas Generales, «la fuerza y la radicalidad de las luchas de las mujeres y su elaboración teórica no habría sido imaginable sin el contexto favorable creado por el 68» (Arruzza, 2015: 61). Mayo desencajó todo de su sitio, también a las mujeres, acaso podríamos decir, especialmente a ellas. Quizá por ello el 68 fue también el inicio del divorcio. La reivindicación del carácter político del sexo y de la sexualidad, contra la centralidad de la producción y de las relaciones de clase, representaba uno de los elementos constitutivos de la separación de los movimientos sociales mixtos operada por el feminismo de los años setenta. Algo también comprensible si tenemos en cuenta cierta cultura asfixiante y paternalista de la que la izquierda nunca supo desembarazarse. El inminente divorcio no implica que en los años siguientes todas las corrientes feministas abandonasen las alianzas y el proyecto de revolución completa de la sociedad en el que se habían implicado. La primera fase del feminismo de segunda ola, que aquí situamos en la década de los setenta y primera mitad de los ochenta ${ }^{4}$, nunca dudó de la necesitad de «transformar las estructuras profundas de la sociedad capitalista» (Fraser, 2015: 252).

Con todo lo dicho podemos afirmar ahora que, paralelamente a la batalla entre Capital-Trabajo, a lo largo de los sesenta discurrieron una serie de luchas que lideraron la exploración de nuevas formas de subjetividad. En esta guerra de guerras, las mujeres, como no podía ser de otro modo, no se quedaron calladas ni quietas. No obstante, los deseos de cambio total despertados en estos años se vieron en buena medida truncados muy poco

4 Atendiendo a la perspectiva de los estudios anglosajones. 
después y una de las razones principales radica en la incapacidad de los movimientos para transformar la matriz de los lazos sociales que daban forma al sistema -en concreto la familia tradicional y la sexualidad dictada por el mercado- en un sentido realmente más liberador a pesar de los duros esfuerzos. Sea como fuere, de las cenizas de Mayo surgiría, de hecho, un «nuevo feminismo» que aspiraba a entrelazarse en ese tronco común de problemáticas.

\section{Los años setenta y ochenta. Entre la interseccionalidad y la identidad}

Si algo distingue a los años setenta es, sin duda, su complejidad, su carácter anfibológico. Década partida que se debatía entre el adiós a la utopía social para recibir a la utopía del capital (de nombre «neoliberalismo») y el intento por expandir el radicalismo vivido, apenas unos años atrás, hacia todos los rincones donde aún no había llegado. En la teoría feminista esta grieta fue perfectamente visible. Los setenta vieron nacer tanto un esfuerzo por recuperar la unión y hacerla aún más efectiva, a partir de la solidaridad de las luchas, como un vuelco del feminismo hacia sí mismo que lo aislaría, ahora sí, como un ismo más en el ecléctico panorama filosófico que por aquel entonces empezaba a conocerse como Posmodernidad 5 .

Nos detendremos ahora en algunos focos de la militancia feminista que estudiaron las relaciones entre la raza, el género y la clase a lo largo de las dos décadas que siguieron a las revueltas de Mayo. Evitaremos hablar de un espacio geográfico preciso ya que las pensadoras y activistas formaron grupos de trabajo internacionales como fue el caso del compuesto por Maria Mies y del tándem que formaron la italiana Mariarosa Dalla Costa y la estadounidense Selma James. A pesar de las diferencias que caracterizan a las propuestas de estas feministas, lo cierto es que en los 70 se produjo un consenso en el seno del movimiento feminista en cuanto a las preguntas que formular para hallar los modelos de opresión que operaban sobre las mujeres. Para Barnet y Philips, «las feministas estaban unidas en la importancia que atribuían a establecer los puntos fundamentales de la causalidad social» (2002, 145). Las diversas causas que contemplaban los distintos pensamientos feministas (socialista, radical o liberal) podían así encontrarse en «el nivel de la estructura social» (142). Bajo este imperativo teórico, veremos en este apartado cómo a principios de los 80 se desarrolla un

5 Como analizaba Seyla Benhabib en su célebre «Feminismo y posmodernidad una difícil alianza», de forma general podemos decir que los distintos feminismos (desde el poscolonial al posestructuralista o el socialista) formaron parte del programa desmitificador de la Posmodernidad y su necesidad de dar muerte a los metarrelatos (como luego harían con los «cuasi-metarrelatos»), tales como el «Sujeto de la Razón», la lógica progresista y teoleológica de la «Historia» o cualquier otra asunción de universalidad. Pero más allá de participar en este tronco común deconstructivo, en los años setenta brotó una serie muy diversa de enfoques feministas, algunos de los cuales entendían que era pertinente realizar un repliegue esencialista o diferencialista. Para un panorama general de este complicado mapa véanse los dos libros-compendio (Nicholson y Amorós). 
movimiento feminista de corte socialista que pretende pensar los problemas de distribución de la riqueza y del trabajo introduciendo el género y la raza como factores decisivos, propuesta que llegó a materializarse en la Conferencia internacional Social-feminista anti-imperialista y anti-racista.

En primer lugar, a mediados de la década del 70, la alemana Maria Mies y su grupo de trabajo abrió una línea original de estudio que estableció una relación estrecha entre la gratuidad del trabajo femenino, la acumulación originaria, la división sexual e internacional del trabajo y las formas de violencia ejercida contra las mujeres. No podemos dejar de mencionar la influencia que tuvieron en Mies los trabajos de Mariarosa Dalla Costa que, junto con Selma James, en el año 1972 publicó The power of women and the subvertion of community, donde arman una sólida crítica de la economía política de explotación de las mujeres. Este camino llevaría, a mediados de los ochenta, hasta hitos como Patriarchy and Accumulation on a World Scale. Women in the International Division of Labour (1986), obra fundamental de la que Silvia Federici se ha declarado deudora en numerosas ocasiones.

Igualmente, durante los primeros años de esta década, activistas y escritoras racializadas respondieron a las inquietudes de multitud de mujeres negras que no se veían representadas dentro de los movimientos feministas de corte socialista. Dieron comienzo así a lo que hoy se ha convertido en la sólida propuesta del feminismo interseccional. Desde el grito de Sojourner Truth «Ain't I a Woman?» en 1851, los principios básicos del feminismo socialista interseccional estaban servidos, sobre todo tras la recuperación que del mismo hizo Selma James, iniciadora de la Campaña Internacional de Salario por el Trabajo Doméstico, en su ya clásico Sexo, raza y clase (1974). Su primer párrafo es más que ilustrador de la tendencia interseccional a la que nos referimos:

Se ha generado mucha confusión cuando el sexo, la raza y la clase se enfrentan por separado e incluso como entidades contradictorias. Que son cosas diferentes es evidente. Que hayan demostrado no estar distanciadas, inseparables, es más difícil de discernir. Sin embargo, si separamos el sexo y la raza de la clase, virtualmente todo lo que nos queda son las políticas truncadas, provincianas y sectarias de la izquierda metropolitana masculina y blanca. Espero mostrar en líneas generales primero, que el movimiento obrero es algo distinto a lo que la izquierda siempre ha promovido. Segundo que, encerrado dentro de la contradicción entre la entidad discreta del sexo o la raza y la totalidad de la clase, está el mayor freno al poder de la clase trabajadora y al mismo tiempo a la energía creativa para conseguirlo (James, 2012: 92).

A lo largo de los setenta, tanto las feministas estadounidenses, como James, Angela Davis, Bell Hooks, Barbara Smith o Patricia Bell Scott, como las inglesas, entre ellas Hazel Carby, Nira Yuval-Davis, Valerie Amos o Pratibha Parmar, recogieron el testigo desafiante 
de la gran oleada de insurrección que había barrido el mundo, precisamente para articular una dura crítica al socialismo masculinizado (Barret y Mclntosh, 1985) y al «feminismo imperialista» (Parmar y Amos, 1984) dando inicio a los estudios raciales. De hecho, en 1982 Smith y Scott editan junto con Gloria T. Hull su ya clásica antología All Women Are White, All the Blacks Are Men, But Some of Us Are Brave. Para varias de estas investigadoras, el fin de sus propuestas era arribar a un nuevo proyecto socialista que incluyese un factor que era ya evidente para todo el mundo, a saber, que la opresión de raza y género interactúa en una red geopolítica compleja con la clase social y con el reparto desigual de la riqueza. Así, en el año ochenta se celebra la Conferencia Social-feminista Anti-imperialista y Anti-racista, en un intento por fundar nuevos foros socialistas internacionales de discusión que abarcasen un análisis interseccional. El objetivo era acercar a los partidos comunistas y socialistas que seguían bajo el diktat del PCUS a esta otra perspectiva. Otro buen ejemplo de esta línea de análisis, lo constituye el texto de las inglesas Margaret Coulson y Kum-Kum Bhavnani «Transformar el feminismo socialista. El reto del racismo» (1986). En él manifiestan la necesidad de que las activistas políticas blancas y las negras trabajen juntas para que el feminismo socialista reconozca una serie de conflictos estructurales tradicionalmente excluidos por el socialismo clásico. En 1986 y refiriéndose al sibilino racismo y sexismo del Estado británico, escriben:

En el primer caso, el Estado abandonó su sexismo de forma negativa, a la vez que consolidaba su racismo al reducir los derechos de mujeres y varones al mismo nivel. En el segundo caso, proclamaba también su compromiso con la igualdad de sexos y mejoraba los derechos de mujeres mayoritariamente blancas, al mismo tiempo que consolidaba su racismo. Así, tras la retórica de la creación de la «igualdad» entre varones y mujeres, el Estado profundiza en las prácticas racistas (Bhavnani y Coulson, 2004: 56).

Llegados a este punto, una pregunta parece obligada: ¿̇qué ocurrió entre la mitad de la década de los 80 y la mitad de la década de los dos mil para que esta fuerza de análisis se fuese apagando paulatinamente, para que estos textos nos lleguen ahora con una frescura inaudita como si ninguna de sus premisas hubiese sido cumplida? Podemos decir que durante una segunda fase de la segunda ola del feminismo los movimientos de mujeres, inspirándose fuertemente en los movimientos afroamericanos, «[extrajeron] el descubrimiento de la diferencia como proceso de afirmación y de diferenciación de la propia identidad» (Arruzza, 2015: 88). En cambio, indujeron, asimismo, al abandono de los factores interseccionales entre clase, raza y género. En los ochenta explosionó una «miríada de pequeños grupos que en la mayor parte de los casos se dedicaron a la práctica de la autoconciencia» (Arruzza, 2015: 88), entorpeciendo el afianzamiento de un feminismo abiertamente interseccional. Encadenado al éxito cre- 
ciente de las filosofías constructivistas posmodernas, todo ello derivó en un indudable desplazamiento del eje de clase en los textos teóricos feministas mayoritarios. Una nueva contradicción o debate se seguirá de esta operación: aquel corte que separa a las políticas de identidad de la reivindicación de la vida de las mujeres en el espacio público y el mundo del trabajo.

\section{4. ¿Éxito o precariedad? El feminismo ante el neoliberalismo}

Algunas teóricas dieron la voz de alarma. Este olvido tenía un precio muy alto. Era en realidad una trampa que llevaba a un oscuro maridaje entre la ideología neoliberal del sujeto «libre» $y$ «hecho a sí mismo» y el ideal de mujer despojada de todas las ataduras -entre ellas las ayudas de un Estado menguante- y preparada para insertarse tanto en el mercado laboral como en los modelos de consumo capitalista que para ella se estaban diseñando. Pero tan pronto como se jugaba tal maniobra, una serie de feministas radicales empezaron a ventilar las incoherencias de lo que denominarían «cultural feminism» y que más tarde conoceremos como feminismo de la diferencia. La principal crítica fue dirigida al proceso de reesencialización de las características y los caracteres de lo femenino y lo masculino en la que parecía inmerso tal «feminismo cultural». En un intento por revalorizar y sacar del absoluto desprestigio en el que se encontraba a todo lo que se asociaba al primero, dibujó un modelo de mujer que, no obstante, sería rápidamente resignificado por el neoliberalismo. Sin contar que el panorama de absoluta separación entre sexos que planteaban podía ser contraproducente cuando no muy peligroso ${ }^{\circ}$.

En su momento sería Nancy Fraser, entre otras, la encargada de hacer de Casandra y querer aguar la fiesta del feminismo liberal. En sus ensayos se esforzó con denuedo en señalar la evolución de los feminismos liberales o su directa fagocitación por el discurso de las elites. Este proceso daría comienzo, según la filósofa política, con ese desplazamiento de las preocupaciones hacia lo cultural que subordinaba el núcleo duro de los conflictos sociales. Si en los primeros pasos del feminismo de segunda ola -aún bajo la influencia o el antagonismo al marxismo-, el interés era ampliar el campo de trabajo y situar las relaciones de género en el terreno de la economía política, en los años ochenta este enfoque parecía agostarse. A juicio de la autora, en un inicio, tanto las tendencias identitarias anglosajonas como las simbolistas francesas no pretendieron suplantar al marxismo sino compensar la inclinación excesivamente economicista de sus teorías pero «nunca dudaron de la importancia de la justicia distributiva y de la crítica a la economía política para el proyecto de emancipación de las mujeres» (Fraser, 
2016: 252). Pero cierto feminismo parecía ahora discurrir por su propia senda hacia la definición de un sujeto mujer psicologicista, homogéneo y simbolicista que se construye casi por sí mismo y con independencia del contexto social. Derivada de una mala lectura de Lacan, esta amalgama de abstracción estructural ahistórica y nostálgica, que hace equivaler tradiciones culturales y estructuras de parentesco con una estructura social, genera una «concepción del orden simbólico que esencializa y homogeniza prácticas y tradiciones históricas contingentes, eliminando tensiones, contradicciones y posibilidades de cambio» (177). Fraser era consciente de la necesidad de apreciar todo aquello que se relacionaba con la labor de las mujeres, pero también de reparar en que lo que ella ha denominado "política de reconocimiento de género» -referida al orden de estatus y a los patrones culturales de interpretación y evaluación codificados socialmente- no debía acabar siendo una mera política de identidad (198). A lo que una política de reconocimiento aspira es a que sea reconocida la identidad de género femenina. La cultura patriarcal desvaloriza y deprecia tal identidad, generando una percepción social devaluada de las mujeres. Una política identitaria implicaría, en tal caso, la remodelación de la identidad que las mismas mujeres tienen sobre sus yoes y la presentación pública de esa remodelación para que sea aceptada por el conjunto de la sociedad (199). Por poner un ejemplo sencillo, la revalorización cultural en una sociedad de las amas de casa, es decir, que una comunidad aprecie su labor, las considere necesarias y ponga en valor su trabajo, no implica un cambio en los niveles de independencia económica o pobreza que estas mujeres adolecen. Fraser aprecia que la lucha por el salario de las amas de casa (redistribución) y la lucha por la valorización social de las mismas (reconocimiento) son distintas pero ambas necesarias. Las segundas estarían destinadas no a valorizar el trabajo de cuidados que realizan las mismas por su valor intrínsecamente femenino, sino, por el contrario, a superar la subordinación a la que están sometidas y que no les permite ser «interlocutoras plenas en la vida social» (200). Quizá sus advertencias no fueron escuchadas. En la década de los 90, junto con el resto de teóricos preeminentes, el grueso de las feministas había abandonado el análisis del mundo del trabajo y de la economía política desde una perspectiva de género. Los estudios de género se convirtieron en una rama más de los programas de Estudios Culturales de las universidades más prestigiosas del mundo. Esto, claro está, tendría un coste. Retrospectivamente, escribe Arruzza:

La opción separatista que ha acompañado en muchos casos la reivindicación del primado de la lucha contra el patriarcado respecto a todas las demás y a la definición de los hombres y de las mujeres como clases de sexos antagonistas, difícilmente ha contribuido a la construcción de una política eficaz a favor de las mujeres. [Sino más bien] al aislamiento y al repliegue sobre sí mismo del movimiento feminista, al desplazamiento hacia la vertiente exclusiva de la crítica ideológica y cultural (2015: 133). 
La historia fue aún más astuta. Trágicamente, este giro del feminismo coincidió con el momento en el que el capitalismo globalizador atacó de forma agresiva cada vez más esferas de la vida, haciendo crecer progresivamente la desigualdad económica tanto local, como global. Es más, durante esta fase se produjo una «inquietante convergencia de algunos de sus ideales con las exigencias de una nueva forma de capitalismo emergente: posfordista, "desorganizada", transnacional» (Fraser, 2016: 244). No es sólo que no se fuese a reconocer económicamente la labor de las mujeres sino que se destacarían sus elementos más precarios para trasladarlos a todos los trabajos. El punto clave de este proceso lo encontramos en el incuestionable hecho de que los trabajadores indispensables para el despliegue del neoliberalismo -tanto asalariados como no asalariados- son desproporcionadamente mujeres. En este período determinadas características de lo doméstico, como la gratuidad, la flexibilidad, etcétera, asaltan el espacio público del mercado laboral, proceso que podemos denominar, con Cristina Morini (2014), housewificación o feminización del trabajo. Así, los feminismos liberales -mayoritarios en Occidente y mayormente blancos-adoptaron y enfatizaron una versión de las mujeres como individuos autosuficientes que cuentan con todas las capacidades que demandaban los empleadores. En lugar de promover una nueva subjetividad basada en la vulnerabilidad y la interdependencia, como más tarde ocurrirá, se quiso equiparar a una parte de la población feminizada con el ideal ilustrado del varón independiente preparado para una carrera de éxitos, mientras se forzaba económicamente a otra parte mucho mayor de las mujeres a ejecutar trabajos asalariados en condiciones de precariedad extrema.

Aún más perverso fue el empeño por trasladar este paradigma de mujer emancipada a países en vías de desarrollo en este preciso momento de expansión del modelo neoliberal a todas las partes del globo. En su excelente pero controvertido artículo «A Dangerous Liason? Feminism and Corporate Globalization», Hester Eisenstein señala las sombrías connivencias entre los programas internacionales de Desarrollo de la Mujer y las estrategias del capital para aumentar la rentabilidad que «han incluido la desindustrialización, la expansión del sector de los servicios y un desplazamiento de la inversión de la producción de bienes a la financiación. Todos estos desarrollos han implicado el uso expandido de la mano de obra femenina», a partir de «una compleja interacción entre un conjunto de estrategias corporativas y gubernamentales para maximizar la rentabilidad, y un movimiento social que buscaba maximizar las opciones de las mujeres, en específico sus oportunidades económicas» (Eisenstein, 2005: 491)7. Esta interacción, como ha señalado Lourdes Benería (2003) generó 
un factor de "oferta» en las nuevas mentalidades de las mujeres y de «demanda» en la preferencia por el trabajo femenino en muchos sectores de la economía. De esta forma, las instituciones financieras internacionales como el Banco Mundial y el Fondo Monetario Internacional crearon Fundaciones Sociales en los países donde se estaban implantando los programas de ajuste estructural y fomentaron de forma masiva los microcréditos y las microinversiones (Elychar, 2002), implicando en esta campaña a Organizaciones No Gubernamentales, Agencias de Desarrollo e instituciones internacionales por la educación (Semple, 2003). En 1997 se celebró la Primera Cumbre Mundial del Microcrédito, llegando a los cien millones de beneficios extraídos de las familias más pobres del globo hacia 2005 (Eisenstein, 2005: 507) por mor de «la emancipación de las mujeres».

En definitiva, podemos decir con Fraser que «el capitalismo contemporáneo no parece necesitar el heterosexismo» (Fraser, 2016: 215), sabe valérselas siempre. Puntualicemos en cualquier caso, antes de cerrar este epígrafe, que nuestro diagnóstico aquí, tomado de la americana, no se dirige al movimiento feminista en su totalidad y diversidad, sino a determinadas ideas arraigadas en el feminismo de corte liberal que coincidieron con los designios del capital durante el período de neoliberalización severa ${ }^{8}$.

\section{Desafíos actuales. Feminismo poscrisis y recuperación de las formas políti- cas del feminismo de los setenta}

¿Qué podemos nosotras extraer de este recorrido? ¿Cuáles son los puntos calientes sobre los que podemos pensar? Con la actual crisis del trabajo, de los cuidados y socioreproductiva que vivimos, en el horizonte stajanovista-neoliberal y posfordista de nuestra actual existencia, en ese telón de fondo donde el capital ha decidido que pagará lo mínimo para la reproducción de la fuerza de trabajo que requiere, a la par que invierte y monetiza cuanto tiene a su alrededor, es decir, en un mundo donde no hay un solo conocimiento, un solo minuto de nuestra vida, un rincón del cuerpo o una actividad humana que no sea productiva, que no sea, trabajo -no siempre remunerado-, el hecho de que hubiese teóricas y activistas subrayando esta realidad nos incita -por no decir, nos apremia- a volver sobre sus textos y actuaciones. Esta es, de hecho, una de las contradicciones sobre la acumulación del capital que David Harvey analiza en Diecisiete contradicciones y el fin del capitalismo: «La reproducción social es para el capital un campo amplio y conveniente en el que los costes reales se externalizan a los hogares y otras entidades comunales, dis- 
tribuyéndose además muy desigualmente entre distintas capas o grupos de la población» (Harvey, 2014: 188). Entre algunas de las causas más dañinas que señala Harvey, se encuentra el endeudamiento personal asociado a la reproducción social, endeudamiento soportado en la mayoría de los casos por mujeres. En el 2001 señalaba Fraser que «los esfuerzos por conseguir una distribución igualitaria están en relativa decadencia» (Fraser, 2016: 190). La realidad es esta.

Tras la crisis económica de 2008, las grandes movilizaciones contra los capitales transnacionales y las bolsas, así como la unión de multitud de activismos como el ecologista, el anticapitalista y el feminista contra los grandes tratados de libre comercio, han encontrado las condiciones para generar eslóganes, propuestas y campañas completas en torno a la cuestión de la redistribución igualitaria de la riqueza y contra las actuales condiciones de reproducción de la fuerza de trabajo ante la situación moribunda de los «estados del bienestar». Fue el caso de la campaña del $99 \%$ versus el $1 \%$ de Bernie Sanders, la Campaña de Unidos Podemos en España en 2016 o la más reciente en Francia liderada por Mélenchon. Es curioso como en el primer y el tercer ejemplo hablamos de líderes políticos que forjaron su activismo político en la década de los 70 . Ocurre de forma similar en los círculos feministas. La recuperación de los primeros textos de Angela Davis, Selma James o Silvia Federici está a la orden del día, pero no en tanto que revival trasnochado sino por lo que sus aportaciones nos siguen tocando. El feminismo parece ser de nuevo el mejor continuador de aquella radicalidad perdida. Las políticas feministas cada vez tienen más presencia en los sindicatos mayoritarios y trabajadoras organizadas del sector servicios han creado sus propias agrupaciones -el sindicato de las Kellys es paradigmático. Creemos que efectivamente en los nuevos movimientos sociales de corte feminista se está produciendo una convergencia entre la lucha feminista por la emancipación y la lucha por la protección social tal y como Fraser la planteó en 2010:

La crítica feminista tendería a rechazar las valoraciones androcéntricas, en especial la sobrevaloración del trabajo remunerado y la infravaloración de los cuidados no remunerados. Presentando los cuidados como un asunto de importancia pública, el movimiento tendería a replantear las soluciones sociales de tal modo que permitiesen a todos efectuar ambos conjuntos de actividades sin las tensiones que hoy en día rodean a tales esfuerzos (Fraser, 2016: 276).

Para que la historia no se repita es conveniente mirar atrás, con la debida precaución, claro es, de no quedarse allí atascado. Uno de los elementos que podemos tomar del pasado -y que de hecho estuvo latente y más o menos visible durante décadas, tal y como hemos comentado- es esa necesidad de conexión de las luchas que fue la clave de bóveda 
del 68 y sus episodios posteriores. Entendemos entonces que el primero de los desafíos es hacer del feminismo un haz de solidaridades otra vez y la cuestión de la distribución y la reproducción se antojan los engarces más inmediatos. El trabajo no pagado inunda el mundo. Parece lógico, entonces, que la reproducción haya vuelto a la palestra, pero la alianza ya no se puede hacer contra los aspectos formales de la producción sino yendo de nuevo a la raíz. Las actividades asociadas a la reproducción siguen siendo un terreno de lucha fundamental para las mujeres «como lo eran para el movimiento feminista de los años setenta» 9 . Cuando pensamos que el trabajo reproductivo no se paga las alianzas se multiplican: de las mujeres con los negros, de los jóvenes precarios con las mujeres, etcétera. Las huellas secretas pero indelebles que la interseccionalidad selló en el feminismo radical son ahora plenamente visibles y quizá también recuperables. El feminismo materialista puso el foco en la división sexual y racial del trabajo desde una perspectiva interseccional amplia. Y habiendo finalmente aprehendido de las enseñanzas de nuestras maestras de los 70, una pregunta, quizá soñadora, puede que descabellada, nos asalta: jes posible imaginar siquiera algo así como una Internacional de las precarias y los no-pagados?

\section{Bibliografía}

Amorós, Celia y Ana De Miguel (coord.) (2005). Teoría feminista: de la ilustración a la globalización. Vol. 2. Del feminismo liberal a la Posmodernidad, Madrid: Minerva.

Arruzza, Cinzia (2015). Las sin parte. Matrimonios y divorcios entre feminismo y marxismo, Madrid: Sylone.

Bantigny, Ludivine (2018). 1968. De grands soirs en petits matins, París: Le Seuil.

Barrett, Michele y Phillips, Anne (2002). Desestabilizar la teoría: debates feministas contemporáneos, Barcelona: Paidós [1992].

Barton, Carol (2004). «Global Women's Movements at a crossroads: Seeking Definition, New Alliances and Greater Impact» en Socialism and Democracy, vol. 18, n¹, pp. 151-184.

BAYNAC, Jacques (2016). Mayo del 68: la revolución de la revolución, Madrid: Acuarela \& A. Machado.

BeneríA, Lourdes (2003). Gender, Development, and Globalization: Economics as if All People Mattered, Nueva York/Londres: Routmedge. 
BÉRARD, Jean (2014). «Dénoncer et (ne pas) punir les violences sexuelles? Luttes féministes et critiques de la répression en France de mai 68 au début des années 1980 » en Politix, vol. 27, N¹07, pp. 61-84.

Bhavnani, Kum-Kum y Coulson, Margaret (2004). «Transformar el feminismo socialista» en Otras inapropiables. Feminismos desde las fronteras, Madrid: Traficantes de Sueños.

Dalla Costa, Mariarosa y JAMEs, Selma (1972). The power of women and the subvertion of community, Bristol: Falling Wall press.

ECHOLS, Carol (1983). «The new feminism of the yin and yang» en Snitow, Ann; Stansell, Christine y Thompson, Sharon (eds.) (1983). Powers of Desire: The Politics of Sexuality, Nueva York: Monthly Review Press.

EISENSTEIN, Hester (2005). «A Dangerous Liason? Feminism and Corporate Globalization» en Science \& Society, vol. 69, № 3, pp. 487-518.

ElyCHAR, Julia (2002). «Empowerment Money: The World Bank, Non-Governmental Organizations, and the Value of Culture in Egypt» en Public Culture, vol. 14, No 3, pp. 493-513.

FederICI, Silvia (2004). Calibán y la bruja. Mujeres, cuerpo y acumulación originaria, Madrid: Traficantes de Sueños.

_ (2013). Revolución en punto cero. Trabajo doméstico, reproducción y luchas feministas, Madrid: Traficantes de Sueños.

FleCKINGER, Helène y Neveux, Oliver (2018). «ll y a de la pensée dans le sexe et du sexe dans la pensée» en Une histoire du spectacle militant (1966-1981), París: L'entretemps éditions.

Fraser, Nancy (2016). Fortunas del feminismo, Madrid: Traficantes de Sueños.

Guattarl, Felix y Negri, Antonio (1974). Las verdades nómadas. Por nuevos espacios de libertad, Donostia: Gakoa Liburuak.

_ (1999). General Intellect, poder constituyente, comunismo, Madrid: Akal.

HARVEY, David (2014). Diecisiete contradicciones y el fin del capitalismo, Madrid: Traficantes de Sueños.

Hull, Gloria T.; Scott, Patricia Bell, y SMITH, Barbara (eds.) (1982). All Women Are White, All Men Black Are Men, But Some of Us Are Brave. Black Women's Studies, Nueva York: The Feminist Press.

James, Selma (2012). Sex, Race, and Class. The Perspective of Winning. A Selection of Writings (1952-201 1), Oakland: PM Press.

Lazzarato, Maurizio y Allez, Éric (2017). Guerres et capital, París: Amsterdam.

MIES, Maria (1986). Patriarchy and Accumulation on a World Scale. Women and the International Division of Labour, Londres: Zed Books. 


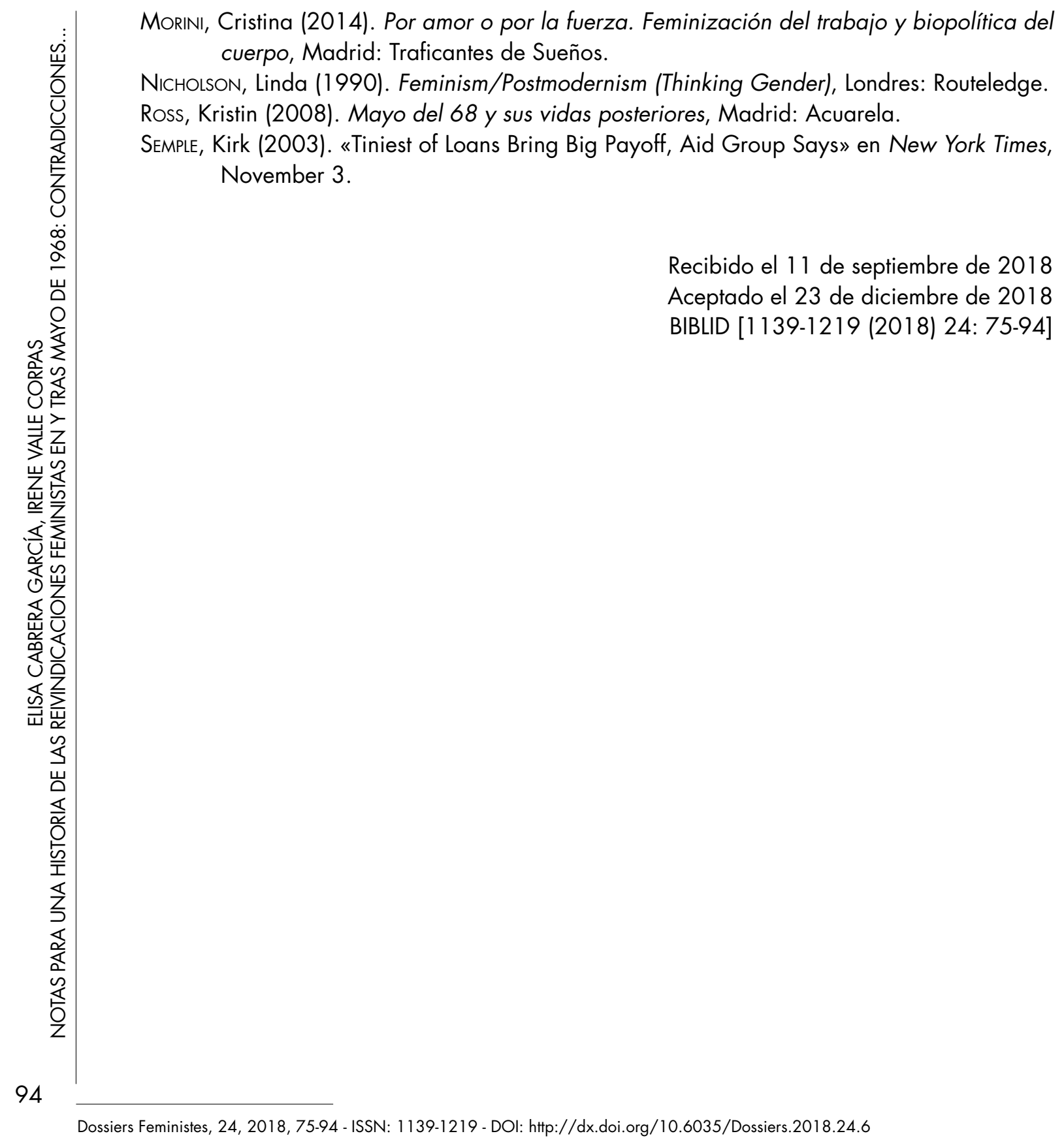

